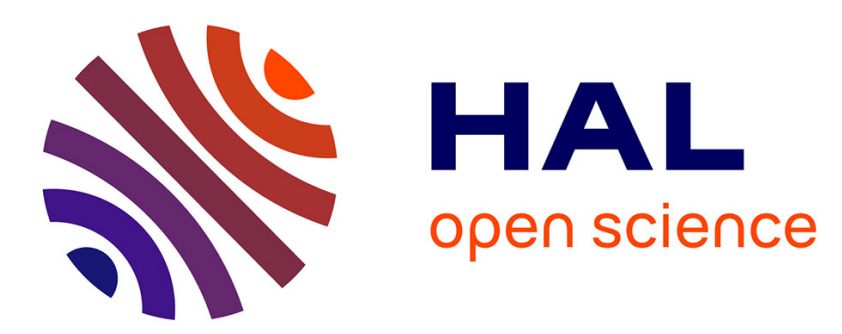

\title{
Demixed and ordered phases in hard-rod mixtures
}

Andras Gabor, Szabolcs Varga, Franz J Vesely, Enrique Velasco, Luis Mederos

\section{To cite this version:}

Andras Gabor, Szabolcs Varga, Franz J Vesely, Enrique Velasco, Luis Mederos. Demixed and ordered phases in hard-rod mixtures. Molecular Physics, 2008, 106 (15), pp.1939-1947. 10.1080/00268970802385253 . hal-00513226

\section{HAL Id: hal-00513226 \\ https://hal.science/hal-00513226}

Submitted on 1 Sep 2010

HAL is a multi-disciplinary open access archive for the deposit and dissemination of scientific research documents, whether they are published or not. The documents may come from teaching and research institutions in France or abroad, or from public or private research centers.
L'archive ouverte pluridisciplinaire HAL, est destinée au dépôt et à la diffusion de documents scientifiques de niveau recherche, publiés ou non, émanant des établissements d'enseignement et de recherche français ou étrangers, des laboratoires publics ou privés. 


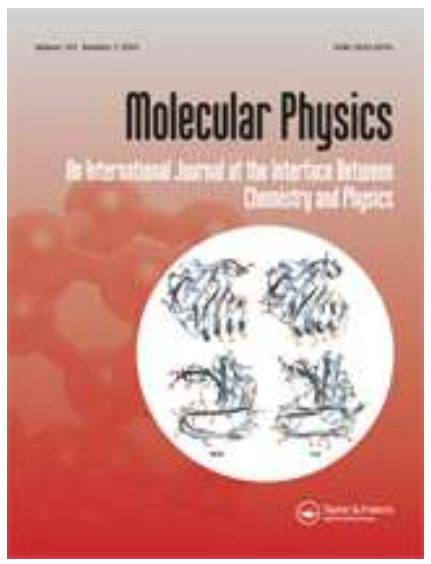

\section{Demixed and ordered phases in hard-rod mixtures}

\begin{tabular}{|c|c|}
\hline Journal: & Molecular Physics \\
\hline Manuscript ID: & TMPH-2008-0147.R1 \\
\hline Manuscript Type: & Full Paper \\
\hline $\begin{array}{r}\text { Date Submitted by the } \\
\text { Author: }\end{array}$ & 28-Jul-2008 \\
\hline Complete List of Authors: & $\begin{array}{l}\text { Gabor, Andras; University of Pannonia, Institute of Physics } \\
\text { Varga, Szabolcs; University of Pannonia, Institute of Physics } \\
\text { Vesely, Franz; University of Vienna, Faculty of Physics } \\
\text { Velasco, Enrique; Universidad Autónoma de Madrid, Departamento } \\
\text { de Física Teórica de la Materia Condensada and Instituto de Ciencia } \\
\text { Mederos, Luis; Consejo Superior de Investigaciones Científicas, } \\
\text { Instituto de Ciencia de Materiales }\end{array}$ \\
\hline Keywords: & liquid crystals, Onsager approximation, smectic ordering, demixing \\
\hline \multicolumn{2}{|c|}{$\begin{array}{l}\text { Note: The following files were submitted by the author for peer review, but cannot be converted } \\
\text { to PDF. You must view these files (e.g. movies) online. }\end{array}$} \\
\hline cylmix_rev.tex & \\
\hline
\end{tabular}

\section{S) ScholaroNE \\ Manuscript Central}




\title{
Demixed and ordered phases in hard-rod mixtures
}

\author{
July 28,2008 \\ Szabolcs Varga, ${ }^{1}$ András Gábor ${ }^{2}$ \\ Institute of Physics, University of Pannonia, H-8201 Veszprém, P.O.Box 158, Hungary \\ Enrique Velasco ${ }^{3}$ \\ Departamento de Física Teórica de la Materia Condensada and Instituto de Ciencia de \\ Materiales Nicolás Cabrera, Universidad Autónoma de Madrid, E-28049 Madrid, Spain \\ Luis Mederos ${ }^{4}$ \\ Instituto de Ciencia de Materiales, Consejo Superior de Investigaciones Científicas, E-28049 \\ Madrid, Spain \\ Franz J. Vesely ${ }^{5}$ \\ Faculty of Physics, University of Vienna, Strudlhofgasse 4, A-1090 Wien, Austria

\begin{abstract}
We analyse demixing and ordering transitions in systems of hard cylindrical particles. The second virial approximation of Onsager and a bifurcation analysis, as introduced by Koda and Kimura, are used to evaluate the free energies, pressures, and density distribution functions in mixtures of equally long but differently wide cylinders. The spatial density distribution along the one relevant coordinate is of particular importance as it provides a more detailed information about the nature of the phase transition than the bare bifurcation diagnosis. Detailed results are given for the nematic-nematic spinodal and the nematic-smectic transitions. Allowing for the absence of an isotropic phase, our results are in good qualitative agreement with those for freely orienting rods reported previously, and indicate a complex sequence of phase diagrams as the diameter dissimilarity of the two components is increased, with upper and lower critical points bounding nematic and smectic demixing regions. However, experimental results on colloidal rods show that nematic demixing occurs at a diameter ratio much smaller than ours or those for freely rotating fluids, indicating that Onsager-type theories may be insufficient to reproduce this phenomenon in a quantitative manner and, consequently, that more sophisticated approaches, presumably incorporating particle flexibility and additional interactions, are required.
\end{abstract}

\footnotetext{
${ }^{1}$ E-mail: vargasz@almos.vein.hu

${ }^{2}$ E-mail: agabor@almos.vein.hu

${ }^{3}$ E-mail: enrique.velasco@uam.es

${ }^{4}$ E-mail: lmederos@icmm.csic.es

${ }^{5}$ E-mail: franz.vesely@univie.ac.at
} 


\section{Introduction}

Fluids of linearly extended particles display an extremely rich phase behavior. In particular, a complex interplay of demixing and spatial ordering may be observed in binary mixtures of rodlike particles. Recent experimental results $[1,2]$ on colloidal rodlike particles which are in qualitative agreement with previous theoretical predictions [3], but differ in quantitative detail, demand a new investigation of such systems. These systems may in principle be modelled approximately by means of hard-core bodies, a type of interaction that has played a crucial role in the theory of liquids and colloids. Even in a system of a single species of hard spherocylinders, all the basic liquid-crystal phases may be produced by simply varying the particle length. In a classic simulation study, Bolhuis and Frenkel [4] scanned the entire phase surface of the one-component hard spherocylinder system. Further simulation studies $[5,6,7,8]$ as well as theoretical investigations $[9,10,11,12,13,14,15]$ followed, leading to an even better understanding of the phase behavior of linear particle systems. In particular, it is now well established that pure hard-body interactions are sufficient to induce spatial ordering transitions in one-component systems, and both demixing and ordering transitions in binary systems.

Numerous new facets arise when we proceed from one-component fluids to mixtures. Koda and Kimura first applied Onsager's second-virial approximation to binary systems of hard spherocylinders of equal diameters but different lengths [16] and to mixtures of spherocylinders and spheres [17]. Dogic et al. [18] introduced arbitrary diameters, and Vesely [19] generalized Koda and Kimura's method to mixtures of variously shaped hard linear particles. Even earlier, Cui et al. [20] had studied a binary system of aligned cylinders in a third-virial approximation. Extensive theoretical studies of freely rotating linear particle mixtures were reported [21, 22, 3, 23, 24, 25, 26, 27, 28, 29]. A multitude of simulation studies followed, both using strictly aligned [30, 31, 32, 18] or freely rotating linear particles [33, 34, 5, 35].

Experience with strictly parallel linear particles has shown that - except for the natural absence of an isotropic phase and the respective phase changes - the main features of systems of freely rotating rods are qualitatively reproduced. Since the numerical approach is generally much less expensive, and some elements of the theory may actually be expressed in closed form, we will here concentrate the discussion to assemblies of parallel rods, although we also present some new results on mixtures of fully rotating hard spherocylinders in order to assess the reliability of the parallel-particle approximation.

We are not the first trying to simplify the molecular model and reproduce the main character of the phase behavior of the system of rod-like particles. Using the Zwanzig approximation for the molecular orientations, several bulk and interfacial properties of the hard rods have been determined [36, 37, 38, 39]. Significant progress has also been achieved in the development of the Onsager theory using the ideas of fundamental measure theory [40]. However the reliability of the new theories and the applicability of the orientational restriction require further theoretical and simulation justification.

The main part of this investigation is a thorough analysis of the phase behaviour of parallel rod mixtures, extending beyond the usual bifurcation analysis. We obtain, at each state point, the true equilibrium state of the system, in particular, the equilibrium density distribution functions of the two particle species, by minimisation of the free energy. In order to do so, we describe the densities of the two species along the $z$ axis, $\rho_{i}(z)$, in terms of a Fourier series, including many more terms than the single cosine term needed in simple bifurcation analyses. In the case of aligned rods, this procedure is quite 
fast and may be extended to 20 or more Fourier terms if necessary. The information gained in this manner permits us a more profound analysis of the phase transitions. For example, at a given point in composition and pressure, the Onsager-Koda stability criterion may indicate a nematic-smectic transition, while the free-energy minimisation reveals that this point is actually lying in the midst of a coexistence region between a nematic and a smectic of different compositions. Several instances of this will be discussed in Section 3.

Two classes of binary systems are interesting candidates for studies of this sort. Rods of equal diameter but different lengths display an intriguing interplay of macro- and microscopic smectic demixing, along with mere smectic layering without demixing. Results on such systems will be reported in a future communication. Here we will discuss particles of equal length but different widths. Since we assume strict alignment along the $z$ axis, the length of the cylindrical particles is just a scaling parameter and does not enter the physical results; the only relevant parameters are the diameter ratio and the number fraction of the mixture. Preliminary reports on such mixtures have already appeared $[27,28]$. Our present results explore a wider range of diameter ratios, thus providing a general picture of phase-equilibria phenomena in these mixtures. According to this picture, as the particle diameters are made more dissimilar, smectic demixing occurs at increasingly lower pressures, eventually interacting with the nematic-smectic phase transition. The manner in which this interaction is realised is quite complex, and an interesting multiphase diagram emerges. Further, nematic demixing may become stable for still larger diameter ratios; this is in general agreement with the findings reported by Van Roij and Mulder [3], but some detailed discrepancies, to be commented later, are apparent. Finally, the scenario that emerges from our model is confirmed by additional calculations in mixtures of rods where the approximation of considering strictly parallel particles is lifted.

The paper is arranged as follows. In the following section we briefly present the model and the approximations used to solve it. Section 3 is devoted to presenting the results of the bifurcation analysis and the phase diagrams that result from the free-energy minimizations. Finally, some conclusions are drawn in Section 4.

\section{Theory}

We consider a binary mixture of parallel thick and thin hard rods where particles are represented as hard cylinders of equal lengths $\left(L_{1}=L_{2}=L\right)$ but of different diameters $\left(D_{1} \neq D_{2}\right)$. Using Onsager theory, we examine the stability of nematic and smectic phases with respect to nematic-nematic, nematic-smectic and smectic-smectic phase separations. As usual, the free energy of the system can be written as a sum of ideal and excess contributions, $F=F_{\text {id }}+F_{\text {ex }}$, where the ideal contribution is given by

$$
\beta F_{\text {id }}\left[\rho_{1}, \rho_{2}\right]=\sum_{i=1}^{2} \int d \mathbf{r} \rho_{i}(\mathbf{r})\left[\ln \rho_{i}(\mathbf{r})-1\right],
$$

while the excess contribution is approximated by the second virial contribution of the virial series of the free energy:

$$
\beta F_{\text {ex }}\left[\rho_{1}, \rho_{2}\right]=-\frac{1}{2} \sum_{i, j=1}^{2} \int d \mathbf{r}_{1} \rho_{i}\left(\mathbf{r}_{1}\right) \int d \mathbf{r}_{2} \rho_{j}\left(\mathbf{r}_{2}\right) f_{\mathrm{M}}^{i j}\left(\mathbf{r}_{12}\right) .
$$


In these equations $\beta=1 / k T$ is the inverse thermal energy and $\rho_{i}(\mathbf{r})$ is the local number density of the components. The Mayer function of the excess contribution is defined as

$$
f_{\mathrm{M}}^{i j}(\mathbf{r})=\left\{\begin{aligned}
-1 & \text { cylinders } i \text { and } j \text { overlap, } \\
0 & \text { otherwise }
\end{aligned}\right.
$$

For the sake of clarity and simplicity, the local number densities are written as a product of mean number density $\rho_{i}=N_{i} / V, i=1,2$, and positional distribution function $f_{i}(z)$, i.e.

$$
\rho_{i}(z)=\rho_{i} f_{i}(z), \quad i=1,2 .
$$

In the nematic phase the probability distribution of the particles is the same everywhere in space. Particles are arranged in a one-dimensional periodic layered structure in the smectic phase, so that the local number densities and positional distribution functions are periodic in the layer spacing $g$. Taking the direction of the density modulation along the $z$ axis, we can write that $\rho_{i}(z)=\rho_{i}(z+g)$, and consequently $f_{i}(z)=f_{i}(z+g)$. As the average of the local densities for one period must be equal to the mean density,

$$
\rho_{i}=\frac{1}{g} \int_{0}^{g} d z \rho_{i}(z),
$$

it is easy to derive the normalisation constraint for the positional distribution function of component $i$

$$
\frac{1}{g} \int_{0}^{g} d z f_{i}(z)=1 .
$$

Therefore the positional distribution functions are equal to unity in the nematic phase, while they exhibit a peaked structure in the smectic phase, with a higher number density on the layers. They are the key quantities in the problem and have to be determined by minimization in order to obtain the equilibrium structure of the system.

Substitution of (4) into (1) and use of the periodic condition (5-6) allows us to obtain the ideal free-energy density as

$$
\frac{\beta F_{\mathrm{id}}\left[f_{1}, f_{2}\right]}{V}=\sum_{i=1}^{2} \rho_{i}\left(\ln \rho_{i}-1+\sigma\left[f_{i}\right]\right),
$$

where

$$
\sigma\left[f_{i}\right]=\frac{1}{g} \int_{0}^{g} d z f_{i}(z) \ln f_{i}(z)
$$

is proportional to the translational entropy. $\sigma$ favours the nematic phase, since it becomes zero for $f_{i} \equiv 1$, while $\sigma>0$ for any positionally dependent $f_{i}$. The excess part of the free energy can be substantially simplified with the help of Eqns. (2-6), which gives the following free-energy density term:

$$
\begin{aligned}
\frac{\beta F_{\mathrm{ex}}\left[f_{1}, f_{2}\right]}{V} & =\frac{1}{2 g} \sum_{i, j=1}^{2} \rho_{i} \rho_{j} \int_{0}^{g} d z_{1} f_{i}\left(z_{1}\right) \\
& \times \int_{z_{12} \in V_{\mathrm{exc}}^{i j}} d z_{2} f_{j}\left(z_{2}\right) A_{\mathrm{exc}}^{i j}\left(z_{12}\right),
\end{aligned}
$$


where $A_{\mathrm{exc}}^{i j}\left(z_{12}\right)$ is the excluded area between two particles of components $i$ and $j$, having a relative displacement $z_{12}$ along the $z$ axis. In the specific case of parallel hard cylinders

$$
A_{\text {exc }}^{i j}\left(z_{12}\right)=\pi\left(\frac{D_{i}+D_{j}}{2}\right)^{2} \Theta\left(L-\left|z_{12}\right|\right) .
$$

The integral of the excluded area gives the excluded volume,

$$
V_{\mathrm{exc}}^{i j}=\int d z A_{\mathrm{exc}}^{i j}(z)
$$

in terms of which the excess free energy of the nematic can be expressed as $\frac{1}{2} \sum_{i j} \rho_{i} \rho_{j} V_{\mathrm{exc}}^{i j}$. It turns out that the nematic excess free energy density can be considerably reduced in the smectic phase because of the peaked density structure, so that Eqn. (10) favours smectic ordering by maximizing the free volume available (packing entropy) for the hard particles. As a result, competition between the translational entropy and the packing entropy terms determines the equilibrium structure of the phase. Note that very similar free-energy expressions can be derived for two-dimensional hard rods on the basis of Onsager theory. The only difference between the two theories is that the smectic period is also a free parameter of the smectic ordering and determined by minimization of the free energy, while the angle that measures the orientation of a rod in a plane is always between 0 and $2 \pi$. If we fix the value of the smectic period $g$, a complete mathematical correspondence can be made between the two theories.

In order to determine the equilibrium structure of the system, the free energy has to be minimized with respect to the positional density distributions and the smectic period. The simplest way to do this is to use a Fourier representation for these functions:

$$
f_{i}(z)=\sum_{j=0}^{n} f_{i j} \cos (j q z)
$$

where $f_{i j}$ is the $j$ th order Fourier amplitude of the distribution function of component $i, q \equiv 2 \pi / g$ is the smectic wavenumber, and $n$ is a cut-off integer. Note that the zerothorder Fourier components are equal to unity because of the normalisation constraint (6). Use of a Fourier representation is advantageous since the orthogonality property of the cosine functions allows for a drastic simplification of the excess free energy. Using Eqns. (10) and (12), and the orthogonality property of the cosine functions, it is possible to derive an analytical expression for the excess free energy:

$$
\begin{aligned}
\frac{\beta F_{\mathrm{ex}}}{V} & =\frac{1}{2} \sum_{i, j=1}^{2} \rho_{i} \rho_{j} \\
& \times\left(V_{\mathrm{exc}}^{i j}+\sum_{k=1}^{n} \hat{A}_{\mathrm{exc}}^{i j} f_{i k} f_{j k} \frac{\sin (k q L)}{k q}\right)
\end{aligned}
$$

where

$$
\hat{A}_{\mathrm{exc}}^{i j}=\pi\left(\frac{D_{i}+D_{j}}{2}\right)^{2} .
$$

In the light of Eqs. $(7,8,12,13)$ and $(14)$, one can see that the free-energy density has the following dependencies

$$
\frac{\beta F}{V}=h\left(\rho_{1}, \rho_{2}, q, f_{11}, f_{21}, \ldots\right)
$$


where $h$ is the sum of (7) and (13). To get the equilibrium density profile and the free energy the following partial derivatives have to be zero at given number densities ( $\rho_{1}$ and $\left.\rho_{2}\right)$

$$
\begin{aligned}
& \frac{\partial h}{\partial f_{1 i}}=0, \quad \frac{\partial h}{\partial f_{2 i}}=0, \quad i=1, \ldots, n \\
& \frac{\partial h}{\partial q}=0 .
\end{aligned}
$$

Having obtained the Fourier components and wavenumber by solving numerically the set of equations (16), the equilibrium free energy density can be determined by substitution of $f_{i j}$ and $q$ into (15). Using the standard thermodynamic relations, the chemical potentials and pressure can be obtained from the free energy as follows:

$$
\begin{gathered}
\beta \mu_{1}=\frac{\partial h}{\partial \rho_{1}}, \quad \beta \mu_{2}=\frac{\partial h}{\partial \rho_{2}}, \\
\beta P=-h+\rho_{1} \frac{\partial h}{\partial \rho_{1}}+\rho_{2} \frac{\partial h}{\partial \rho_{2}} .
\end{gathered}
$$

The first non-vanishing results of the set of equations (16) for $f_{i j}$ and $q$ give the nematic-smectic bifurcation density and wavenumber. From the result of this bifurcation analysis, the curves of the second-order nematic-smectic phase transition can be drawn. However there is no guarantee that the phase transition between nematic and smectic phases is of second order. In addition, no information can be gained from the bifurcation analysis as far as the stability of nematic-nematic and smectic-smectic demixing transitions are concerned. To make a complete analysis it is necessary to search for demixing phase transitions in the nematic and smectic phases and for first order phase transitions between nematic and smectic phases. If a first-order transition between two phases does occur, the coexisting densities can be obtained from the pressure and chemical potential conditions. Denoting the coexisting phases as $\alpha$ and $\beta$, the requirement is the equality of the pressures and chemical potentials of each components in both phases, i.e. $P_{\alpha}=P_{\beta}$, $\mu_{1 \alpha}=\mu_{1 \beta}$, and $\mu_{2 \alpha}=\mu_{2 \beta}$.

\section{Results}

In this section we present our bifurcation and Fourier-expansion results for the phase diagrams of binary mixtures of parallel thin and thick hard cylinders. In addition, to check the effect of orientational freedom, the Parsons-Lee density functional theory (PLDFT) has also been applied to a mixture of freely rotating hard spherocylinders. The reason for using a different particle shape in the freely rotating case is that the determination of the overlap region for a cylinder capped with a hemisphere in both ends is much easier than for cylinders, and in any case the hemispheric ends do not significantly affect the ordering behaviour of the system (we do not include the PL-DFT equations here as the theory has been fully presented in earlier publications; see e.g. [28]).

Since all particles have identical lengths, and the properties of systems of parallel particles are invariant under length scaling along the director, the only free parameter of the Onsager theory is the diameter ratio between the particles of the two components. In the following the thin cylinders will be labelled by 1 , while the thick ones have label 
2. We define the diameter ratio as $d=D_{2} / D_{1}$, which therefore is larger than one always. We present our results in terms of dimensionless quantities, such as reduced pressure $P^{*}=\beta P v_{1}$, packing fraction $\eta=N_{1} v_{1} / V+N_{2} v_{2} / V$, and reduced wave number $q^{*}=q L$ and smectic period $g^{*}=g / L$, where $v_{i}$ is the particle volume of the $i$-th species.

The bifurcation analysis from the nematic phase allows for a quick overview of how the nematic-smectic bifurcation line evolves with composition and diameter ratio. Thus, we first determined the bifurcation packing fraction, pressure and wave number of the nematic-smectic bifurcation line as a function of composition $x=N_{1} / N$ and $d$. The resulting surfaces are presented in Fig. 1. Since both components have the same length, the resulting wave number (hence smectic period) does not depend on either of the above parameters, and universal values $q^{*}=4.4934$ and $g^{*}=1.3983$ are obtained at the bifurcation point. However, Fig. 1 shows that the packing fraction and the pressure strongly depend on the actual values of composition and diameter ratio (note that the Onsager theory predicts in some cases unphysical values of the packing fraction; this shortcoming may be cured by using density scaling, e.g. along the lines of Parsons and Lee, but it does not qualitatively change the predictions of the Onsager theory). Starting from the pure phase of either thick or thin rods, the bifurcation packing fraction decreases as particles of the other species are added to the monodisperse system, i.e. enhanced stability of the smectic phase can be achieved by mixing thin and thick rods. This effect can be seen very clearly in the case of a sea of thin rods, where addition of only a tiny amount of thick rods can substantially decrease the bifurcation density. In the other end of the bifurcation diagram, addition of thin rods to the fluid of thick rods has just a slightly decreasing effect on the packing fraction. The reason for this is that thick rods give rise to a much higher excluded-volume cost in the sea of thin rods than the other way round. To maximize the free volume available, particles have to arrange themselves in layers, and this occurs at a much lower packing fraction in the first case.

The enhanced stability of the smectic with respect to the nematic is more pronounced as the volume difference between the thin and thick cylinders is increased. Since the bifurcation analysis is only reliable for second-order phase transitions, the determination of the bulk properties of the smectic phase is crucial to take into account the possible firstorder transitions between nematic and smectic phases and demixing transitions between two smectic phases. Solving the set of equations for the Fourier components and wave number, Eqn. (16), and equating the pressure and chemical potentials to obtain the coexisting phases, we have found that the bifurcation analysis gives the right transition densities up to $d=2.67$. For higher diameter ratios, it is found that the nematicsmectic transition becomes first order. Also, in the high-pressure regime, a smecticsmectic demixing transition occurs, as we presently show.

Fig. 2(a) shows a typical phase diagram of a mixture of thin and thick rods where both first order nematic-smectic transitions and smectic-smectic demixing transitions can be seen. Interestingly, two smectic demixing transitions are observed; one is closed down by a lower critical point, while the other is bounded by an upper critical point. In the inset of Fig. 2(a), we show how the nematic-smectic two-phase region becomes larger in composition and pressure as the diameter ratio is increased from $d=2.67$ to 2.70 . The nematic-smectic transition becomes first order in the thin-rod rich part of the phase diagram, where the unfavourable thin-thick hard-body interaction is dominant. It can also be seen that a small region of smectic-smectic demixing, closed by a upper critical point, develops from the nematic-smectic biphasic region for $d=2.7$. As a result, a smectic-smectic-nematic triple point appears in the phase diagram (note that, at the 


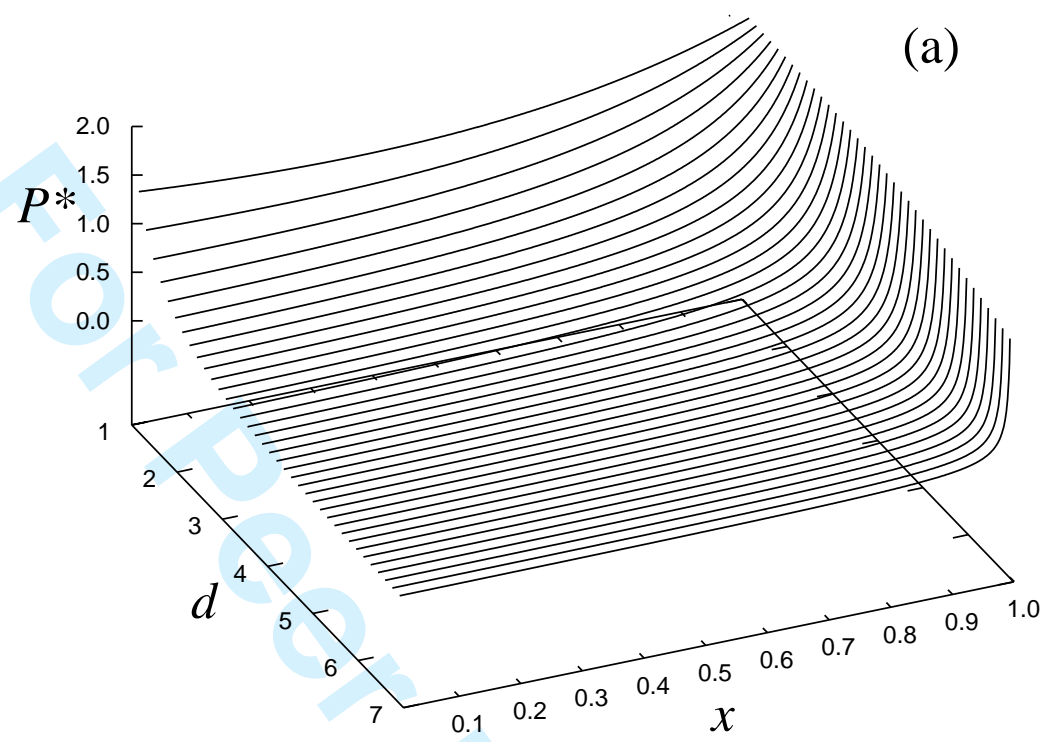

(b)

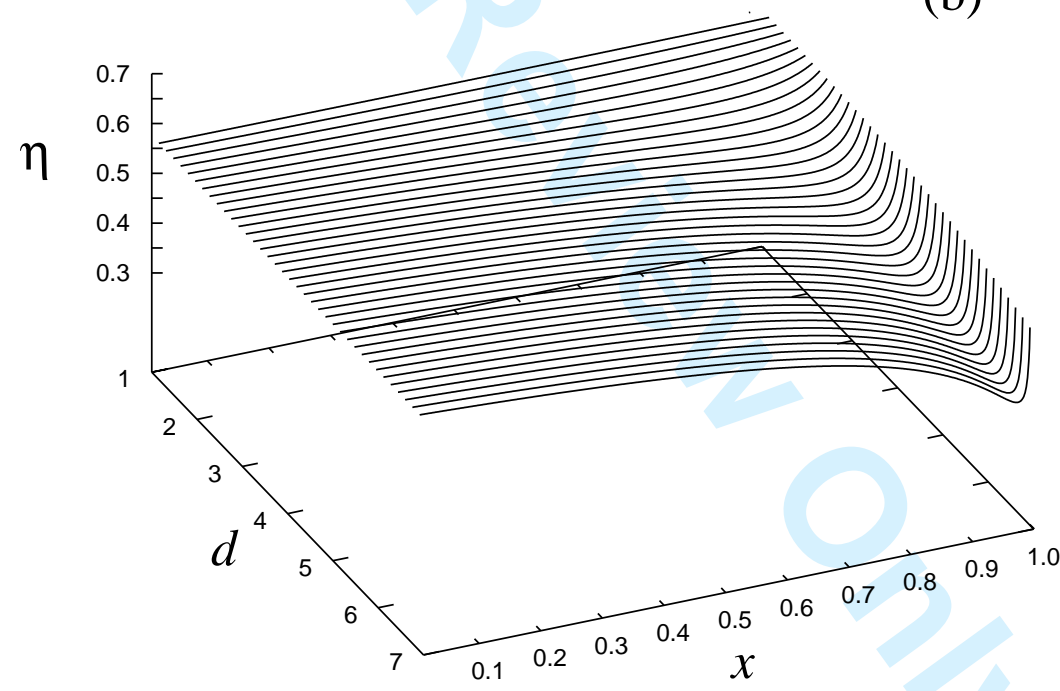

Figure 1: Nematic-smectic bifurcation packing fraction (a), and pressure (b) of binary mixtures of parallel thin and thick hard rods as a function of composition $x$ and diameter ratio $d$. The surfaces are the boundary between nematic and smectic phases. $x$ corresponds to the mole fraction of the thin component. 

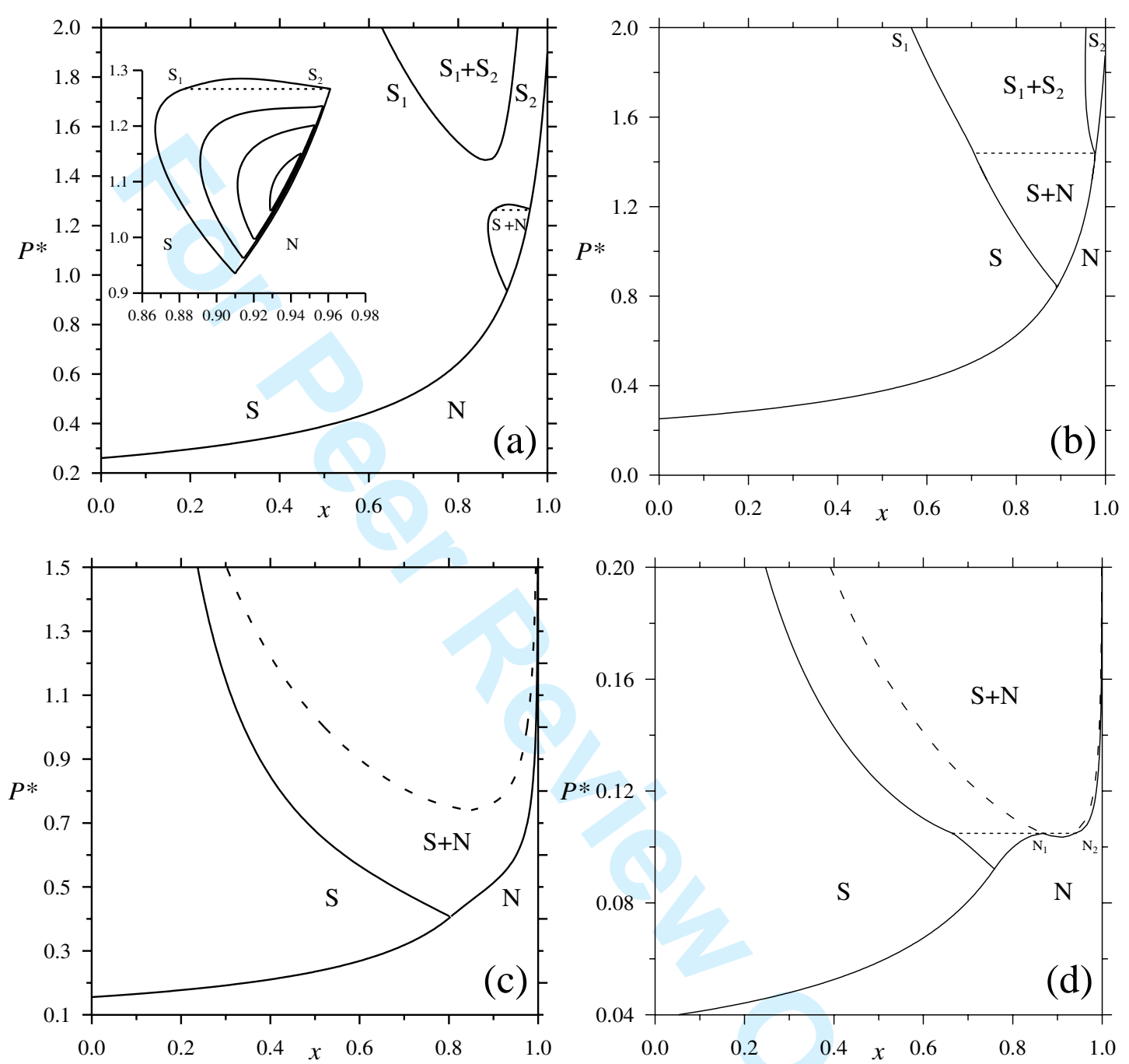

Figure 2: Pressure-composition $\left(P^{*}-x\right)$ phase diagrams for mixtures of parallel thin and thick hard rods with diameter ratios $d=2.7$ (a), 2.75 (b), 3.5 (c) and 7 (d). The labels $\mathrm{N}_{1} \mathrm{~N}_{1}, \mathrm{~N}_{2}, \mathrm{~S}, \mathrm{~S}_{1}, \mathrm{~S}_{2}$ denote the nematic and smectic phases, respectively. $x$ corresponds to the mole fraction of the thin rods. The horizontal short-dashed line indicates the three-phase coexistence pressures in (a), (b) and $(\mathrm{d})$, while the dashed curve shows the unstable nematic-nematic demixing transition in (c) and (d). The inset in (a) shows the effect of varying diameter ratio on the stability of nematic-smectic and smectic-smectic phase separations. The values of the diameter ratios in the inset from inside to outside are: $d=2.67,2.68,2.69$ and 2.70 . 
point, two of the phases are critical, so that the point should more properly be called a critical end point).

As $d$ is increased, a sudden change in the topology of the phase diagram appears. Both critical points collapse and the segregation regions coalesce. This can be seen in Fig. 2(b), where no upper and lower critical exist, but a single nematic-smectic-smectic three-phase line (again two of the phases are critical) divides the nematic-smectic and the smectic-smectic phase transitions for the system with $d=2.75$. Our estimates indicate that the lower smectic-smectic critical point, which exists whenever these two coexistence regions occur as separate phenomena, does so for $d<2.705$, whereas the upper critical point appears only in the small interval $2.695<d<2.705$.

Similar phenomenon has been found in the nematic region of the phase diagram of the mixture of freely rotating thin and thick rods using Onsager type theories [27]. Disappearance of the lower critical point has been observed experimentally [1], too. Sudden change of the phase topology can be observed in other types of systems such as the binary mixture of platelets [41].

At this point it is worthwhile mentioning that the topology of the phase diagram of two-dimensional binary mixtures of freely rotating hard rectangles [42] can be identical and show the same trends with increasing size ratio as the three-dimensional mixtures studied here. Making the change of variable $\psi=2 \pi z$ in the integral over the $z$ variable, the free energy density (15) can be rewritten as a function of $\psi$, with $0<\psi<2 \pi$. Since the orientation of a two-dimensional object can be characterized by the orientational distribution function $h(\phi)$, where the angle $\phi$ range in the interval 0-2 $\pi$, one could say that our positional distribution function $f(z)$ may be identified with an orientational distribution function. The nematic phase of our three-dimensional particles corresponds to the isotropic phase of two-dimensional particles, while the smectic phase can be associated with the nematic phase.

The topology of the phase diagram does not change too much with increasing size difference. The two-phase regions become wider and the smectic phase is more and more stable with respect to the nematic phase. These trends are shown in Fig. 2(c) for $d=3.5$. For this diameter ratio, experiments demonstrate that a (purely repulsive) binary mixture of colloidal particles consisting of fd- and PEG-coated fd-viruses shows a stable first-order nematic-nematic demixing transition [1]. In an earlier study [27] it was pointed out that this nematic demixing transition can be explained at the level of Onsager theory using the perfect alignment approximation. However, the possibility of smectic ordering and, therefore, of transitions from nematic to smectic phases, were not included in those calculations. Results from the present study show that the nematicnematic transition [dashed curve in Fig. 2(c)] is preempted by the transition between nematic and smectic phases for this value of the diameter ratio. In order to find a stable nematic-nematic demixing transition, it is necessary to extend our analysis to larger diameter ratios, which are certainly beyond the range of experimentally feasible diameter ratios in the above colloidal mixture. We found that the first binary mixture exhibiting a stable nematic-nematic demixing transition is given by $d \simeq 7$ [see Fig. 2(d)] . The transition becomes stable for mixtures rich in the thin component, for which the unlike excluded-volume interaction is the strongest.

As we have seen, as the diameter ratio $d$ is increased, smectic demixing at high pressure becomes stable at decreasing values of pressure; this demixing region is bounded below by a lower critical point [Fig. 2(a)]. At the same time, as $d$ increases the firstorder nematic-smectic transition becomes wider in composition and eventually develops 
a region of smectic demixing bounded by an upper critical point. The two critical points eventually collapse into a single one to give an increasingly wider segregation region [Fig. $2(\mathrm{~b}-\mathrm{d})]$. This mechanism does also occur when the orientational degrees of freedom of the rods are not frozen. Fig. 3 shows a phase diagram pertaining to freely-rotating hard spherocylinders for the case $d=2.38$. The inclusion of orientational entropy in these mixtures is therefore essentially irrelevant in the demixing mechanism involving nematic and smectic phases, save the change of order from second to first in the bulk nematic-smectic phase transition of the pure components (which persists as a first-order transition for the mixture in the whole composition interval). The reason behind this is the relatively high value of the nematic order parameter at the pressure range where demixing phenomena are occurring.

The above results show that the interplay between nematic-smectic and nematicnematic phase separations in mixtures of parallel thick and thin rods is indeed very important. However, a very high size ratio is required to stabilize the nematic-nematic demixing transition, which causes a highly fractionated nematic-smectic transition; this latter feature is missing in the experimentally observed phase diagrams of the $\mathrm{fd}+\mathrm{fd}-$ PEG colloidal system. The discrepancy between theory and experiment cannot be traced to the most obvious simplifications of the theoretical models; in particular, as we have seen, similar phase diagrams are obtained irrespective of the inclusion of orientational 
degrees of freedom in the model. This may imply that mixtures of fd and fd-PEG viruses cannot be modelled as hard bodies. Additional interactions that discourage the formation of smectic order are needed in the model. We believe such interactions may come from the chiral character of interactions in fd viruses and from their flexibility.

\section{Conclusions}

The phase behaviour of a binary mixture of thin and thick rods has been examined using Onsager and rescaled Onsager theories. Using the restriction of perfect orientational alignment, i.e. frozen orientational degrees of freedom, both a bifurcation analysis from the nematic phase and the parametric minimization of the free energy show that the mixture undergoes a continuous phase transition between nematic and smectic phases for $d<2.67$, irrespective of the composition. For larger diameter ratios, the nematicsmectic transition becomes of first order when the mixture is rich in thin rods. The coexisting smectic phase is richer in thick rods than the nematic phase, which reflects the incompatible hard-body interactions between thick and thin rods, and also that sufficient entropy can be gained by arranging thick rods in a layered structure. The unfavourable thin-thick interaction becomes more pronounced for higher diameter ratios, where the biphasic region of nematic-smectic coexistence gets wider and, in addition, gives rise to smectic demixing, with one phase rich in thick rods and the other in thin rods. Smectic segregation becomes more pronounced as pressure (density) is increased, because in this way packing effects are optimized, even at the cost of decreasing the mixing entropy.

The topology of the phase diagram is identical to that of freely rotating two dimensional binary mixture of hard rods since in both two- and three-dimensional systems lower and upper critical points terminate the demixing transitions for low values of $d$, and three-phase coexistence takes place for high $d$. The only differences between the twoand three-dimensional systems is that, in the latter case, nematic and smectic phases correspond, respectively, to isotropic and nematic phases in two dimensions, and that all transitions are of first order in two dimensions.

Inclusion of the orientational freedom in the theory shows that the orientational entropy does not affect qualitatively either the properties of smectic phases or the smectic demixing transition. However orientational entropy broadens the biphasic regions, makes the nematic-smectic phase transition be of first order, and gives rise to the isotropicnematic phase transition. To justify the usefulness of the perfect-alignment approximation and the simple Onsager theory, we plan to extend our calculations to mixtures of short and long rods. The experimentally observed nematic-nematic demixing transition has been observed only for very high diameter ratios $(d>7)$ in the perfect alignment approximation or in the full model with freely orienting particles. To obtain nematicnematic demixing at lower diameter ratios, close to the experimental values $d \simeq 3$, we believe that flexibility effects and Coulomb interactions have to be explicitely included in the model. We think that further steps can be achieved along this way on the basis of earlier studies of the system of slightly flexible hard rods [43], and that of charged colloidal rods [44].

SV, AG and FV would like to thank the financial support of the Austrian-Hungarian Action Foundation (grant number: 68öu1). Support from the Hungarian-Spanish (HH20062005) and Austrian-Spanish (HU2006-0006) Integrated Actions Programmes, and grants S-0505/ESP-0299 from the Comunidad Autónoma de Madrid, and FIS2005-05243-C0201, FIS2005-05243-C02-02, and FIS2007-65869-C03-01 from the Ministerio de Educación 
y Ciencia of Spain, are also acknowledged.

\section{References}

[1] K. R. Purdy, S. Varga, A. Galindo, G. Jackson and S. Fraden, Phys. Rev. Lett. 94, 057801 (2005).

[2] M. Adams, Z. Dogic, S. L. Keller, and S. Fraden, Nature 393, 349 (1998).

[3] R. van Roij and B. M. Mulder, Phys. Rev. E 54, 6430 (1996).

[4] P. Bolhuis and D. Frenkel, J. Chem. Phys. 106, 666 (1997).

[5] M. P. Allen, G. T. Evans, D. Frenkel, and B. Mulder, Adv. Chem. Phys. 86, 1 (1993).

[6] J. Crain and A. V. Komolkin, Adv. Chem. Phys. 109, 39 (1999).

[7] D. Costa, F. Micali, F. Saija, and P. V. Giaquinta, J. Phys. Chem. B 106, 12297 (2002).

[8] S. C. McGrother, D. C. Williamson, and G. Jackson, J. Chem. Phys. 104, 6755 (1996).

[9] B. Mulder, Phys. Rev. A 35, 3095 (1987).

[10] J. D. Parsons, Phys. Rev. A 19, 1225 (1979).

[11] S. D. Lee, J. Chem. Phys. 87, 4972 (1987); J. Chem. Phys. 89, 7036 (1988).

[12] A. Poniwierski and R. Holyst, Phys. Rev. Lett. 61, 2461 (1988).

[13] M. P. Taylor, R. Hentschke, and J. Herzfeld, Phys. Rev. Lett. 62, 800 (1989).

[14] A. M. Somoza and P. Tarazona, J. Chem. Phys. 91(1), 517 (1989).

[15] A. M. Somoza and P. Tarazona, Phys. Rev. A 41, 965 (1990).

[16] T. Koda and H. Kimura, J. Phys. Soc. Jap. 63, 984 (1994).

[17] T. Koda, M. Numajiri, and S. Ikeda, J. Phys. Soc. Jap. 65, 3551 (1996).

[18] Z. Dogic, D. Frenkel and S. Fraden, Phys. Rev. E 62, 3925 (2000).

[19] F. J. Vesely, Mol. Phys. 103, 679 (2005).

[20] S. Cui and Z. Y. Chen, Phys. Rev. E 50, 3747 (1994).

[21] A. Samborski, G. T. Evans, C. P. Mason, and M. P. Allen, Mol. Phys. 81, 263 (1994); A. Samborski and G. T. Evans, J. Chem. Phys. 101, 6005 (1994).

[22] P.C.Hemmer, J. Stat. Phys. 100, 3 (2000).

[23] R. van Roij, B. M. Mulder, and M. Dijkstra, Physica A 261, 347 (1998).

[24] H. Bosetti and A. Perera, Phys. Rev. E 63, 021206 (2001).

[25] H. N. W. Lekkerkerker, Ph. Coulon, R. Van Der Haegen, and R. Deblieck, J. Chem. Phys. 80, 3427 (1984).

[26] S. Varga, A. Galindo and G. Jackson, Mol. Phys. 101, 817 (2003).

[27] S. Varga, K. R. Purdy, A. Galindo, S. Fraden, and G. Jackson, Phys. Rev. E 72, 051704 (2005). 
[28] G. Cinacchi, Y. Martínez-Ratón, L. Mederos, and E. Velasco, J. Chem. Phys. 124, 234904 (2006).

[29] G. Cinacchi, Y. Martínez-Ratón, L. Mederos, and E. Velasco, Mol. Cryst. Liq. Cryst. 465, 121 (2007).

[30] A. Stroobants, H. N. W. Lekkerkerker, and D. Frenkel, Phys. Rev. Lett. 57, 1452 (1986).

[31] T. Koda and S. Ikeda, Mol. Cryst. Liq. Cryst. 318, 101 (1998).

[32] T. Koda, Y. Sato, and S. Ikeda, Prog. Th. Phys. Suppl. 138, 476 (2000).

[33] P. Bolhuis and D. Frenkel, J. Chem. Phys. 101, 9869 (1994).

[34] M. Dijkstra and R. Van Roij, Phys. Rev. E 56, 5594 (1997).

[35] X. Zhou, H. Chen, M. Iwamoto, J. Chem. Phys. 120, 1832 (2004).

[36] Y. Martínez-Ratón and J.A. Cuesta, J. Chem. Phys. 118, 10164 (2003).

[37] Y. Martínez-Ratón, Phys. Rev. E 69, 061712 (2004).

[38] M. Bier, L. Harnau, and S. Dietrich, Phys. Rev. E 69, 021506 (2004).

[39] Y. Martínez-Ratón, et al., in New Approaches to Old and new Problems in Liquid State Theory, proceedings of the NATO-ASI (Series C) 529, 139 (1999).

[40] Y. Martínez-Ratón, et al., Phys. Rev. E 77, 051205 (2008).

[41] L. Harnau, D. Rowan and J.-P. Hansen, J. Chem. Phys. 117, 11359 (2002).

[42] D. de las Heras, Y. Martínez-Ratón, and E. Velasco, Phys. Rev. E 76, 031704 (2007).

[43] P. van der Schoot, J. Phys. II France 6, 1557, (1996).

[44] H.H. Wensink, J. Chem. Phys. 126, 194901 (2007). 


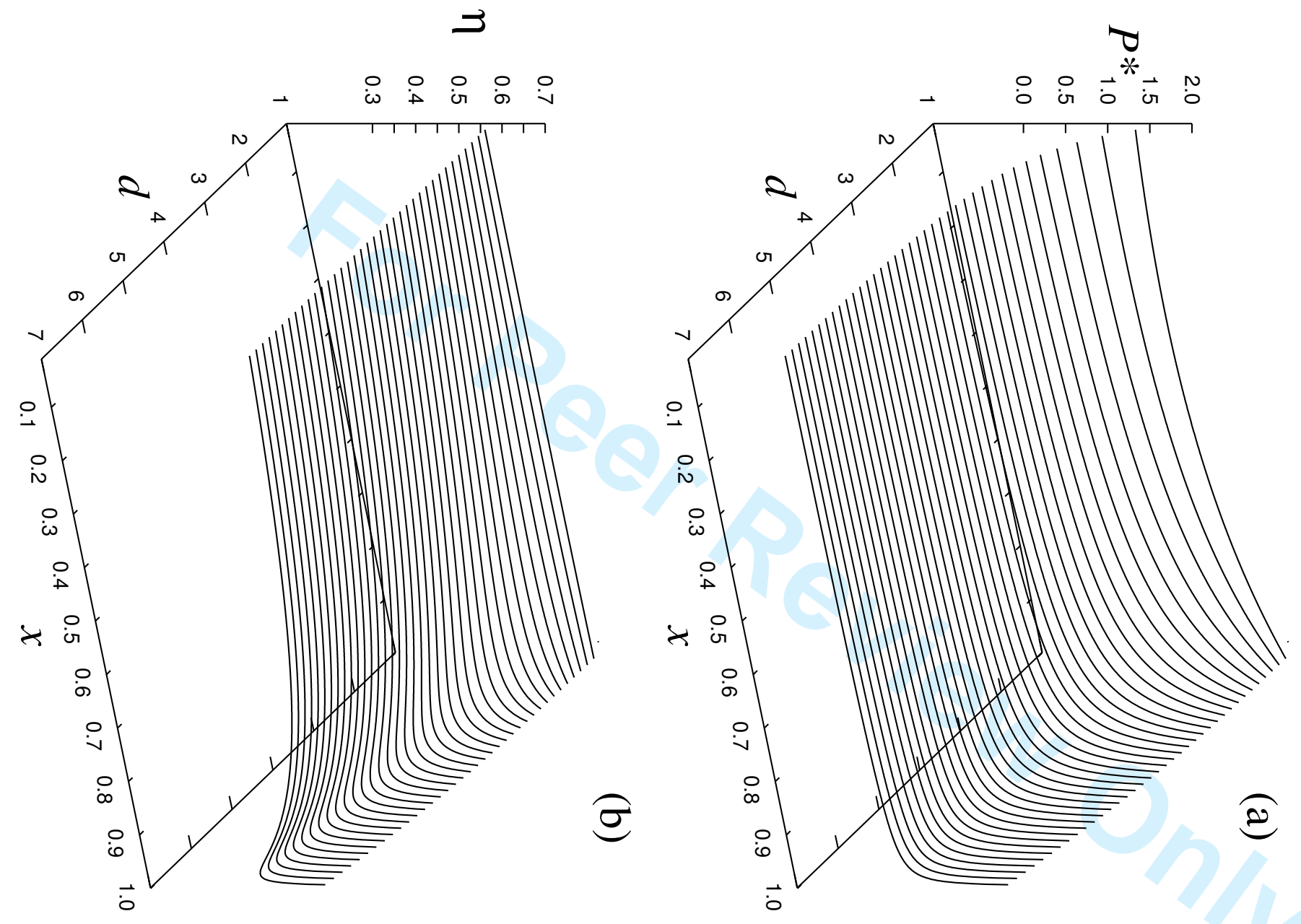




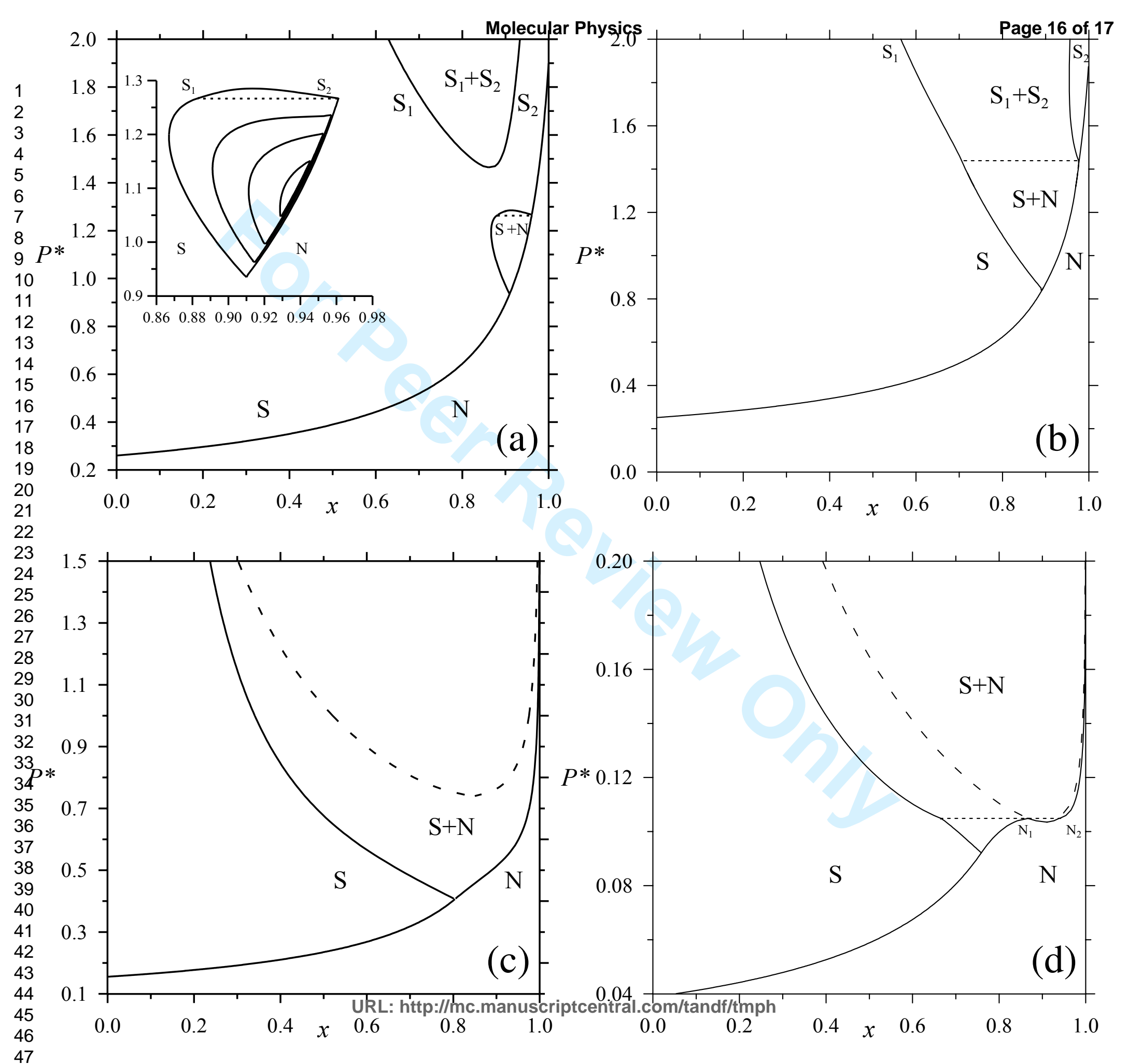


Page 17 of 17

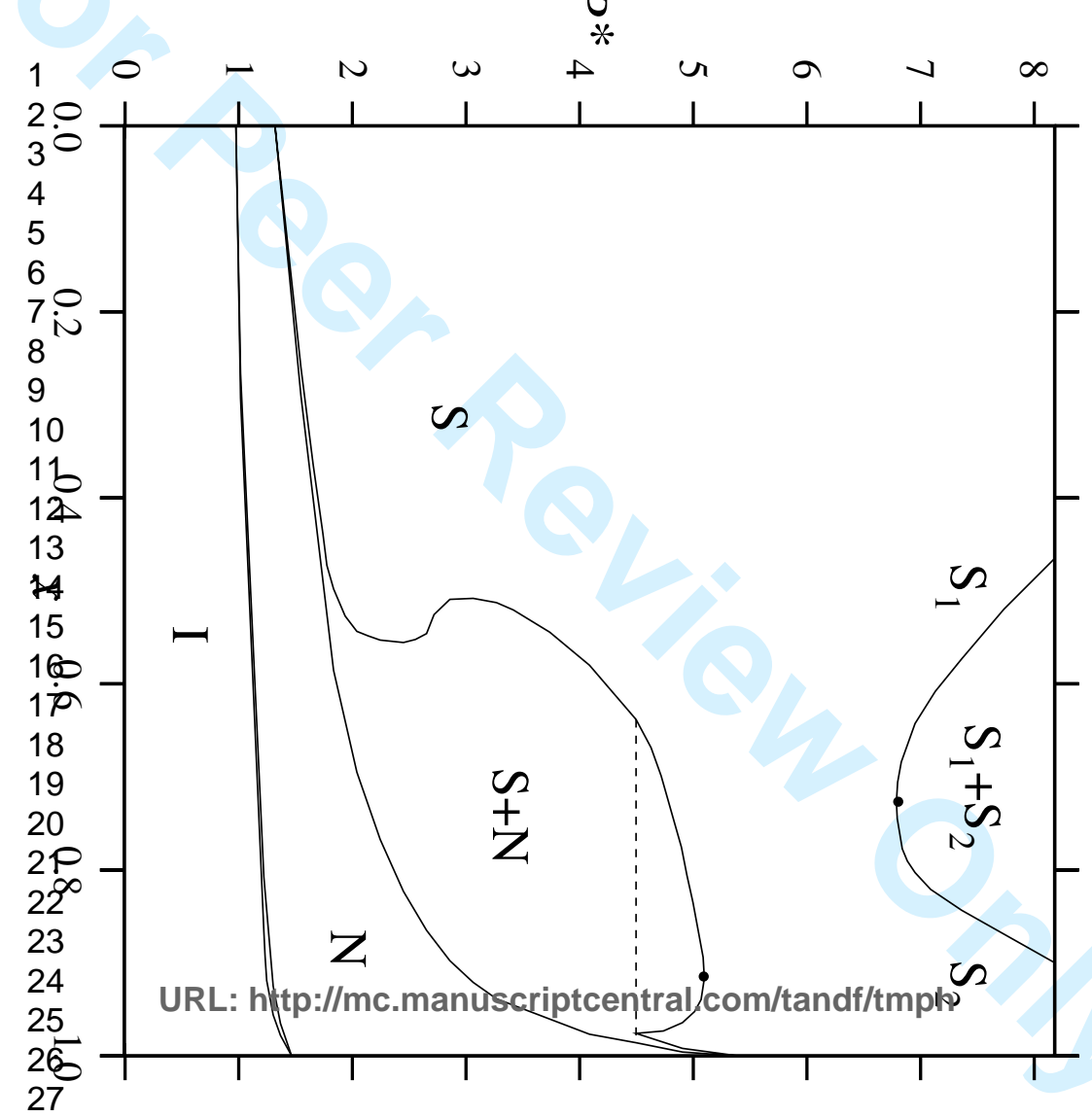

